\title{
20-Hydroxyeicosatetraenoic acid regulates the expression of Nedd4-2 in kidney and liver through a neddylation modification pathway
}

\author{
JIANZHU ZHAO ${ }^{1,2}$, BIJUN ZHANG $^{1}$, GUANGRUI LAI ${ }^{1}$, RUNHONG XU $^{1}$, GUOMING CHU $^{1}$ and YANYAN ZHAO ${ }^{1}$ \\ ${ }^{1}$ Department of Clinical Genetics; ${ }^{2}$ Oncological Department, \\ Shengjing Hospital of China Medical University, Shenyang, Liaoning 110004, P.R. China
}

Received July 10, 2016; Accepted August 18, 2017

DOI: $10.3892 / \mathrm{mmr} .2017 .7803$

\begin{abstract}
The present study aimed to test whether 20-hydroxyeicosatetraenoic acid (20-HETE) affected neddylation modification of E3-ligase Nedd4-2 (neural precursor cell expressed, developmentally down-regulated 4-like, E3 ubiquitin protein ligase). A cytochrome P450 family 4 subfamily F member 2 (CYP4F2) transgenic mouse model that overproduces 20-HETE in the kidney and the liver was used in the present study. Transgenic mice with high salt intake exhibited increased activation of Nedd4-2-mediated ubiquitin-proteasome pathway. Nedd4-2 expression is increased in the kidney and decreased in the liver of transgenic mice compared with wild-type mice. Subsequently, co-immunoprecipitation analysis indicated that Nedd4-2 was modified by Nedd8, and the level of neddylation on Nedd4-2 was reduced in the kidney and increased in the liver of transgenic mice compared with controls. In addition, sentrin-specific protease 8 (Senp8), a deneddylation enzyme, is expressed higher in the kidney and lower in the liver of transgenic mice compared with wild-type controls. The function of 20-HETE on modulation of Nedd4-2 were also confirmed in mouse M1 kidney and mouse NCTC1469 liver cell lines, and the function was restored by neddylation inhibitor MLN4924 Data from the present study demonstrated that 20-HETE upregulated the expression of Nedd4-2 in the kidney and downregulated expression in the
\end{abstract}

Correspondence to: Professor Yanyan Zhao, Department of Clinical Genetics, Shengjing Hospital of China Medical University, 36 Sanhao Street, Heping, Shenyang, Liaoning 110004, P.R. China E-mail: yyzhao@sj-hospital.org

Abbreviations: 20-HETE, 20-hydroxyeicosatetraenoic acid; Nedd4-2, neural precursor cell expressed developmentally downregulated 4-like, E3 ubiquitin-protein ligase; CYP4F2, cytochrome P450 family 4 subfamily F member 2

Key words: 20-hydroxyeicosatetraenoic acid, neddylation, Nedd4-2, sentrin-specific protease $8, C Y P 4 F 2$ transgenic mice liver through the neddylation modification pathway, at least partly, depending on the effects on Senp8 deneddylation.

\section{Introduction}

20-Hydroxyeicosatetraenoic acid (20-HETE) is primarily produced in the kidney and the liver, and serves a role in hypertension, metabolic dysfunction and cancers. It has been reported previously that 20-HETE functions in a tissue-specific manner, particularly in hemorheology, which may account for its 'prohypertensive' function in peripheral arteries like pulmonary arteries (1) and its 'antihypertensive' role in coronary arteries (2). Our previous study developed a cytochrome P450 family 4 subfamily $\mathrm{F}$ member 2 (CYP4F2) transgenic mouse that overproduced 20-HETE in kidney and liver, exhibiting a hypertension phenotype (3). Our previous study also demonstrated that Nedd4-2-mediated ubiquitin-proteasome pathway was induced in the transgenic mice with high salt intake, which resulted in the degradation of kidney-specific $\mathrm{Na}-\mathrm{K}-\mathrm{Cl}$ symporter2 (NKCC2), and thus affected natriuresis and blood pressure (4). Therefore, the present study hypothesized that production of the multifunctional 20-HETE metabolite in a tissue-specific pattern may be related to its role in modulating Nedd4-2.

As an E3 ubiquitin ligase of the Nedd4 family, Nedd4-2 probably modulates and binds to multiple membrane proteins to aid in their internalization and turnover $(5,6)$. Dysfunctions of Nedd4-2 have been implicated in many human pathologies through the targeted membrane proteins; for example, inhibition of the interaction between Nedd4-2 and epithelial sodium channel (ENaC) leads to Liddle's syndrome (7), downregulation of voltage-gated sodium channels by Nedd4-2 contributes to neuropathic pain (8), altered levels of Nedd4-2 are linked to multiple tumor types $(9,10)$ and genetic variation in Nedd4-2 is associated with hypertension (11). Therefore, the present study examined post-translational modifications to explore the potential mechanism of 20-HETE-regulated Nedd4-2 expression in the $C Y P 4 F 2$ transgenic mouse model.

Neddylation is a post-translational modification whereby the ubiquitin-like protein, Nedd8, is conjugated to target proteins. Like the ubiquitin pathway, neddylation is a cascade pathway of specific E1, E2 and E3 enzymes, which deliver 
Nedd 8 to substrates in a covalent bond. So far defined, the only E1 that is involved in the neddylation process is a heterodimer comprising amyloid $\beta$ precursor protein-binding protein 1 and ubiquitin-like modifier-activating enzyme3. There are two E2 enzymes involved in the neddylation process: Ubiquitin-conjugating enzyme E2 $\mathrm{M}$ and ubiquitin-conjugating enzyme E2 F $(12,13)$. Certain enzymes have been reported to be of the E3 type in neddylation, such as regulator of cullins 1/RING-box protein 1 (12-14) and Smurf1 (15). This post-translational modification was reported to be involved in an extensive pathophysiology process depending on the specific E3, and the best studied of these E3 ligases belong to the cullin-RING ubiquitin ligase (CRL) family (16). Neddylation has been reported to promote the ubiquitination activity of CRLs for the activation of cullin 3-adaptor-E3 ubiquitin ligase complex and the prevention of binding to the structural-based inhibitory factor (17). Neddylation of CRLs may be reversed through deneddylation, a process that removes Nedd8 through the isopeptidase activity of the COP9 signalosome complex (CSN) (18). Therefore, both neddylation and deneddylation, which cycle E3 ligases between activated and inactivated states, may be essential for the ubiquitination activities of E3 ligases. Furthermore, an inhibitor of neddylation E1, MLN4924, has been reported to stop the neddylation cycle (19) and to effectively suppress the development of multiple tumor types $(20,21)$. As aforementioned, it was reported that HECT-type E3 ubiquitin-protein ligase Smurf1 was activated by neddylation through a thioester bond at $\mathrm{C} 426$, which was revealed to be correlated with colon cancer progression and poor prognosis (15).

The present study aimed to elucidate the neddylation of Nedd4-2 and to determine the tissue-specific influence of 20-HETE on Nedd4-2. Nedd4-2 expressions in the liver and the kidney were compared between $C Y P 4 F 2$ transgenic mice and wild-type mice, and the interaction of Nedd4-2 with Nedd8 was examined. The results may provide the first evidence that 20-HETE regulated Nedd4-2 through neddylation modification in kidney and liver.

\section{Materials and methods}

Antibodies and reagents. Rabbit anti-Nedd8 polyclonal antibody was obtained from Enzo Life Sciences, Inc. (catalog no. ALX-210-194-R200; Farmingdale, NY, USA); rabbit anti-Senp8 polyclonal antibody was obtained from Abcam (catalog no. ab58423; Cambridge, UK); rabbit anti-Nedd4L polyclonal antibody (catalog no. 13690-1-AP); rabbit anti-tubulin polyclonal antibody (catalog no. 10094-1-AP) and mouse anti-GAPDH monoclonal antibody (catalog no. 60004-1-Ig) were purchased from ProteinTech Group, Inc. [(Chicago, IL, USA). Fetal bovine serum (FBS) and RPMI-1640 culture medium were purchased from Biological Industries, USA, Inc. (Cromwell, CN, USA)], and 20-HETE was purchased from Cayman Chemical Company (catalog no. 90030; Ann Arbor, MI, USA). MLN4924 was purchased from Active Biochem (A-1139; Kowloon, Hong Kong).

Transgenic mouse. This study was approved by the Ethics committee of Shengjing Hospital (Shenyang, China). All animal-related experiments conformed to the Guide for the
Care and Use of Laboratory Animals published by the US National Institutes of Health (NIH Publication no. 85-23, revised 1996). The CYP4F2 transgenic mice overexpressing CYP4F2 was a FVB strain (3). Experiments were performed on 12- to 16-week-old male transgenic mice weighing 24-30 g. All mice were matched in weight and age with littermate wild-type FVB mice as controls (a gift from Animal Laboratory of China Medical University). All mice were provided with food and water ad libitum under a 12-h light-dark cycle and controlled temperature $\left(23 \pm 2^{\circ} \mathrm{C}\right)$.

Cell culture. The mouse M1 kidney cell line and the mouse NCTC1469 liver cell line were purchased from the Type Culture Collection of the Chinese Academy of Sciences (Shanghai, China) and were cultured in DMEM and RPMI-1640 medium, respectively, at $37^{\circ} \mathrm{C}$ in a humidified $5 \% \mathrm{CO}_{2}$ atmosphere. Cells were cultured in 6-well plates until $75 \%$ confluence, after which the cells were placed in fetal bovine serum-free medium (99\% DMEM or 1640 with $1 \%$ mycillin) for $4 \mathrm{~h}$ at $37^{\circ} \mathrm{C}$ in a humidified $5 \% \mathrm{CO}_{2}$ atmosphere. Control cells were treated with vehicle ( $0.1 \%$ ethanol). Experimental cell cultures were treated with 20-HETE at different concentrations $(0.1,0.5,1$, 2.5 or $10 \mu \mathrm{M}$ for $1 \mathrm{~h}$ at $37^{\circ} \mathrm{C}$ in a humidified $5 \% \mathrm{CO}_{2}$ atmosphere), followed by incubation with $1 \mu \mathrm{M}$ 20-HETE for 10, 30, 60,120 or $240 \mathrm{~min}$ in a $37^{\circ} \mathrm{C}$ incubator. The greatest change of protein expression in Nedd4-2 occurred at $1 \mu \mathrm{M}$ at $120 \mathrm{~min}$. Subsequent analyses were performed under these conditions. The medium was replaced with medium containing $10 \%$ fetal bovine serum and $1 \%$ mycillin $30 \mathrm{~min}$ prior to harvest. For MLN4924 treatments, the cells were treated with MLN4924 at different concentrations $\left(0.1,1\right.$ or $2.5 \mu \mathrm{M}$ for $24 \mathrm{~h}$ at $37^{\circ} \mathrm{C}$ for $120 \mathrm{~min}$ after $1 \mu \mathrm{M} 20$-HETE was added). The greatest change in protein expression of Nedd4-2 was observed with $2.5 \mu \mathrm{M}$ MLN4924 treatment, and subsequent experiments were performed under these conditions.

Immunohistochemical staining. Mice were sacrificed by decapitation. Their kidney and liver were quickly removed and then fixed in $10 \%$ formalin solution for $48 \mathrm{~h}$ at room temperature. The tissues were subjected to routine processing for paraffin embedding, and sections $(4 \mu \mathrm{m})$ were placed onto glass slides coated with triethoxysilane, air-dried, fixed in a $50 \%$ acetone/methanol solution at $4^{\circ} \mathrm{C}$ for $2 \mathrm{~h}$, deparaffinized with xylene, rehydrated in an alcohol series and washed with PBS. Some of the sections were stained with hematoxylin and eosin for $3 \mathrm{~min}$ at room temperature to determine the histological type. For the remaining sections, antigen retrieval was performed in $10 \mathrm{mM}$ sodium citrate buffer $(\mathrm{pH} \mathrm{6}$ ) for $10 \mathrm{~min}$ at $100^{\circ} \mathrm{C}$, then the sections were subjected to blocking of endogenous peroxidases by incubation for $30 \mathrm{~min}$ in $3 \%$ $\mathrm{H}_{2} \mathrm{O}_{2}$ solution at room temperature, and blocking non-specific binding sites with $1 / 100$ diluted goat serum for $40 \mathrm{~min}$ at room temperature. After blocking, the sections were incubated with primary antibodies $(1: 100)$ at $4^{\circ} \mathrm{C}$ overnight. Sections were washed with PBS and incubated with biotin-conjugated secondary antibodies for $30 \mathrm{~min}$. The sections were washed with PBS and avidin-biotin complex $(1: 1,000)$ was added to the sections for $60 \mathrm{~min}$ at room temperature. Following extensive washing with PBS, immunoreactive products were visualized by reaction with 3,3'-diaminobenzidine, catalyzed 
by horseradish peroxidase in the presence of $\mathrm{H}_{2} \mathrm{O}_{2}$. Sections were counterstained with Gill's hematoxylin for $3 \mathrm{~min}$ at room temperature, dehydrated in ascending methanol series, cleared with xylene and mounted under a coverslip. All Images were observed using light microscope (Nikon Eclipse CI; Nikon Corporation, Tokyo, Japan).

Western blot analysis. Total protein was extracted from cells (6 well plates, $2.5 \times 10^{6}$ cells in each well) or tissues (weighing $150 \mu \mathrm{g}$ ) and the concentration was determined with the Bradford method. Proteins $(40 \mu \mathrm{g})$ were denatured at $95^{\circ} \mathrm{C}$ for $\geq 5 \mathrm{~min}$ and were separated by one-dimensional $10 \%$ SDS-PAGE and transferred onto polyvinylidene fluoride (PVDF) membranes (catalog no. ISEQ00010; EMD Millipore, Billerica, MA, USA). Membranes were subsequently blocked with skimmed milk powder [5\%, diluted in $1 \%$ TBS containing $0.5 \%$ Tween-20 (TBST)] at room temperature for $2 \mathrm{~h}$. Then the PVDF membrane was washed with TBST. After that the membrane was incubated with primary antibodies against Nedd4-2 (1:1,000), Nedd8 (1:1,000), Senp8 (1:1,000), tubulin $(1: 10,000)$ or GAPDH $(1: 10,000)$ at $4^{\circ} \mathrm{C}$ overnight with gentle shaking. Membranes were incubated with horseradish peroxidase-conjugated goat anti-rabbit IgG (catalog no. ab97051; 1:5,000) or goat anti-mouse IgG (catalog no. ab97023; 1:5,000) (both from Abcam) for $>1 \mathrm{~h}$ at room temperature. Following washing with TBST, protein bands were visualized with an Enhanced Chemiluminescence kit (catalog no. B18005; Biotool; Selleck Chemicals, Shanghai, China). Densitometry was performed using Quantity One software version 4.2 (Bio-Rad Laboratories, Inc., Hercules, CA, USA) and normalized to GAPDH or tubulin.

Co-immunoprecipitation (co-IP). Kidney and liver tissue (weighing $800 \mu \mathrm{g}$ ) were prepared by homogenizing the frozen tissues or cells in four $75 \mathrm{~cm}^{2}$ cell culture flasks in lysis buffer containing protease inhibitors, and the concentration was determined by the Bradford method. A total of $500 \mu \mathrm{g}$ protein was incubated with Nedd4-2 primary antibody (1:500, catalog no. 13690-1-AP, ProteinTech Group, Inc. Chicago, IL, USA) rotating at $4^{\circ} \mathrm{C}$ for $3 \mathrm{~h}$ overnight. Subsequently, protein A/G PLUS-agarose (30 $\mu \mathrm{l}$; Santa Cruz Biotechnology, Inc., Dallas, TX USA) was added and incubated with rotation at $4^{\circ} \mathrm{C}$ overnight. The protein $\mathrm{A} / \mathrm{G}$ beads were then collected by centrifugation $\left(4^{\circ} \mathrm{C}, 2,200 \mathrm{x} \mathrm{g}, 5 \mathrm{~min}\right)$ and washed three times with TBST. Immunoprecipitates were incubated with 2X loading buffer (Beijing TransGen Biotech, Co., Ltd., Beijing, China) and immediately denatured $95^{\circ} \mathrm{C}$ for $>5 \mathrm{~min}$ and analyzed by western blotting.

Reverse transcription-quantitative polymerase chain reaction $(R T-q P C R)$. Total RNA $(1 \mu \mathrm{g})$ was extracted from tissues $(50 \mu \mathrm{g})$ and cells ( 6 well plates, $2.5 \times 10^{6}$ cells in each) using TRIzol reagent (Invitrogen; Thermo Fisher Scientific, Inc., Waltham, MA, USA), and was reverse transcribed with random primers using the Reverse Transcription Reagent kit (Takara Biotechnology Co., Ltd., Dalian, China)), following the manufacturer's protocol. qPCR was performed using SYBR Premix Ex Taq II (catalog no. RR820; Takara Biotechnology Co., Ltd.), according to the manufacturer's protocol, and the Applied Biosystems 7500 Real-Time PCR
System (Thermo Fisher Scientific, Inc.). The qPCR reaction condition was as follows: $95^{\circ} \mathrm{C}$ for $5 \mathrm{~min}$; followed by 40 cycles of $95^{\circ} \mathrm{C}$ for $15 \mathrm{sec}$ and $60^{\circ} \mathrm{C}$ for $50 \mathrm{sec}$. As an internal control, GAPDH was measured under the same conditions in RT-qPCR. Primer sequences were: Nedd4-2, forward 5'-TGAAGCCCAATGGGTCAGAAATA-3', reverse 5'-GGA CCCTGTTCACAAATCTCCAC-3'; and GAPDH, forward 5'-GAAGGTGAAGGTCGGAGTC-3', reverse 5'-GAAGAT GGTGATGGGATTTC-3'. mRNA expression levels were quantified using the $2^{-\Delta \Delta \mathrm{Cq}}$ and normalized to the internal reference gene GAPDH (22).

Statistical analysis. All experimental data were derived from at least three independent experiments. All expression data were presented as mean \pm standard error of the mean. Statistical analysis of two samples was performed with Student's t-test analysis. Statistical analyses among multiple groups were performed using a one-way analysis of variance followed by Bonferroni's post hoc test. Analysis was performed using SPSS 17.0 (SPSS, Inc., Chicago, IL, USA). P $<0.05$ was considered to indicate a statistically significant difference.

\section{Results}

Comparison of Nedd4-2 expression between CYP4F2 transgenic mice and wild-type mice. As 20-HETE is produced mainly in the kidney and liver, the present study aimed to determine the effects of 20-HETE on Nedd4-2 expression in kidney and liver tissues by comparing the expression levels of Nedd4-2 between $C Y P 4 F 2$ transgenic mice and wild-type control mice. Western blotting results demonstrated that Nedd4-2 protein is expressed at higher levels in kidney and lower levels in liver of transgenic mice compared to expression in the matching tissues of wild-type mice (Fig. 1A). Immunohistochemical analysis confirmed that Nedd4-2 protein expression was higher in transgenic kidney tissues and lower in transgenic liver tissues compared with the wild-type control tissues (Fig. 1B). Conversely, RT-qPCR results identified no significant differences between the Nedd4-2 mRNA expression levels in kidney and liver of transgenic mouse compared with wild-type mice (Fig. 1C). Similarly, no significant differences were identified between the Nedd4-2 mRNA expression levels in mouse kidney cell line M1 and mouse liver cell line NCTC1469 when 20-HETE was added (Figs. 1D and 4B). These results indicated that 20-HETE was able to regulate Nedd4-2 expression in a tissue-specific manner, and this regulation was at the post-transcriptional level.

Nedd4-2 is neddylated in kidney and liver tissues. As neddylation is an important post-translational modification of E3 ligase, whether or not Nedd4-2 is modified by Nedd8 was examined. Co-IP was used to determine the interaction between Nedd 8 and Nedd4-2 in kidney and liver tissues, which demonstrated that Nedd4-2 was modified by Nedd8 in both kidney and liver tissue (Fig. 2A). However, Nedd8 binding to Nedd4-2 was weaker in kidney and stronger in liver of transgenic mice compared with wild-type controls, which was opposite to protein expression in kidney and liver. In addition, Nedd8 protein expression was detected in kidney and liver tissues by immunohistochemistry (Fig. 2B). Nedd8 was expressed higher in both liver and kidney tissues of transgenic mice compared with expression in 
A
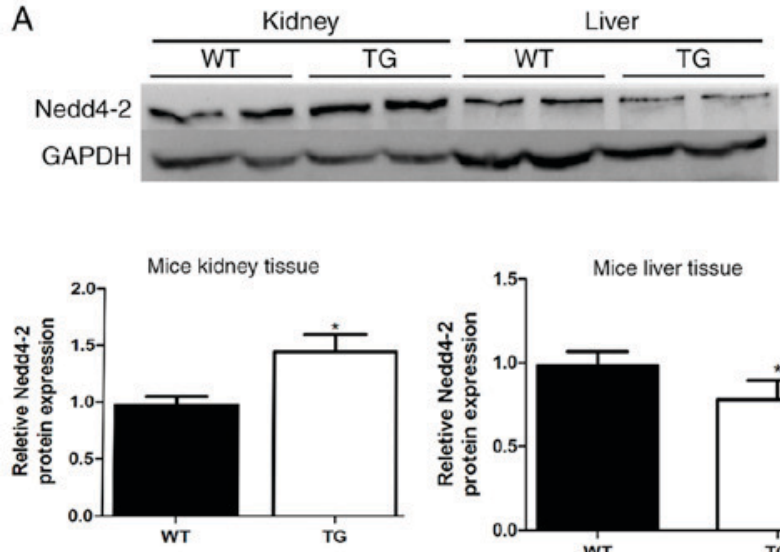

C

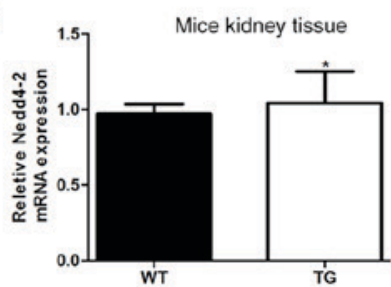

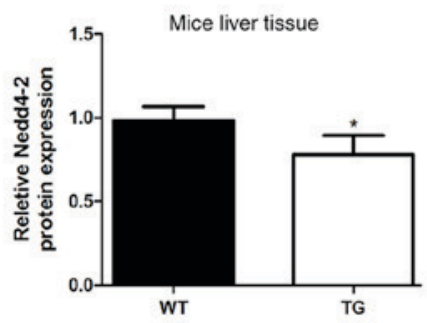

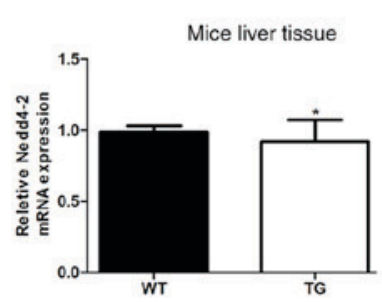

B
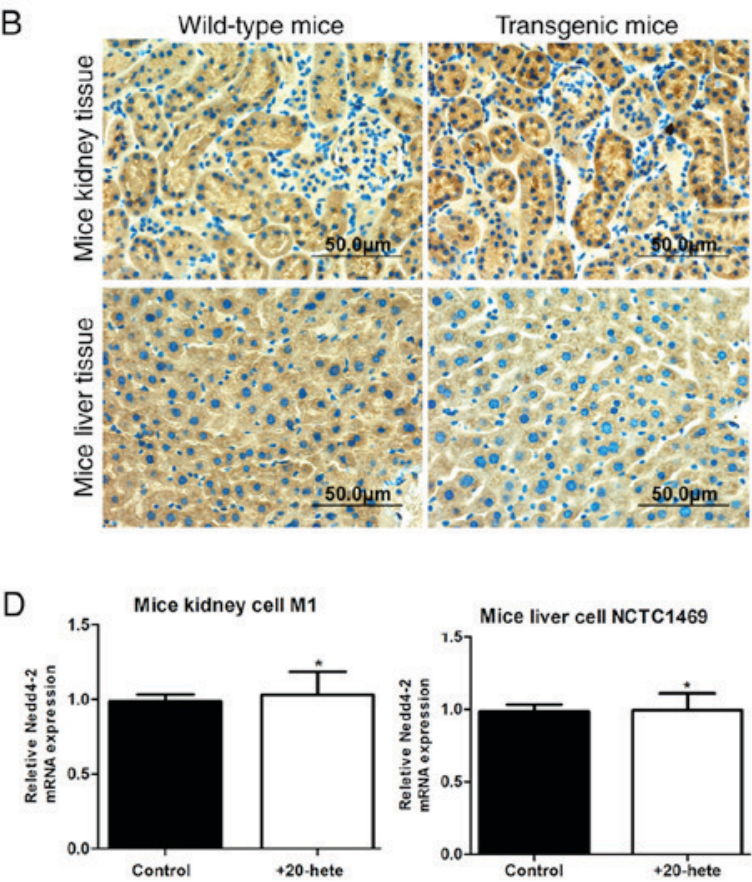

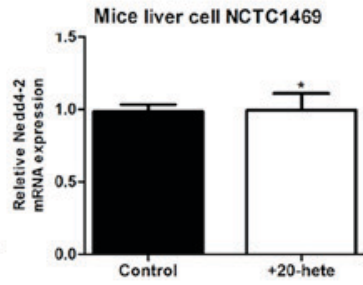

Figure 1. Expression of Nedd4-2 in kidney and liver tissues of transgenic mice and wild-type controls. (A) Western blotting revealed a relatively higher level of Nedd4-2 protein expression in kidney and a lower level in liver of transgenic mice compared with wild-type mice. All experiments were performed three times independently; $\mathrm{n}=6$ (the tissues in these 3 independent experiments were from 6 mice including 3 TG mice and $3 \mathrm{WT}$ mice); $\mathrm{P}<0.05$ vs. WT kidney; " $\mathrm{P}<0.05$ vs. WT liver. (B) Representative immunostaining images of the expression of Nedd4-2 in kidney and liver tissues; Scale bar, $50 \mu$ m; $\mathrm{n}=6$; (C) Reverse transcription-quantitative polymerase chain reaction analyses indicated no significant difference of Nedd4-2 mRNA expression in kidney and liver of transgenic mice and wild-type controls. (D) Reverse transcription-quantitative polymerase chain reaction analyses indicated no significant differences were identified between the Nedd4-2 mRNA expression levels in mouse kidney cell line M1 and mouse liver cell line NCTC1469 when 20-HETE was added. All experiments were performed three times independently. Nedd4-2, neural precursor cell expressed developmentally downregulated 4-like, E3 ubiquitin-protein ligase; TG, transgenic; WT, wild-type.

A

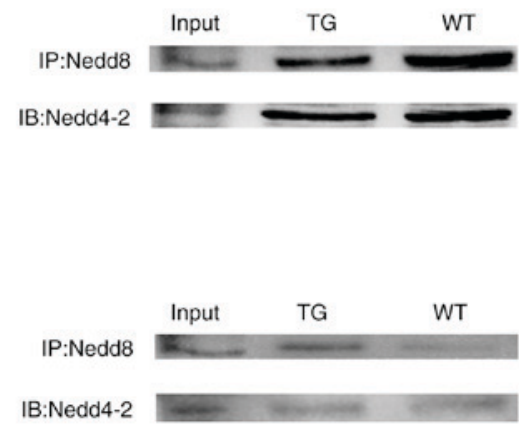

B

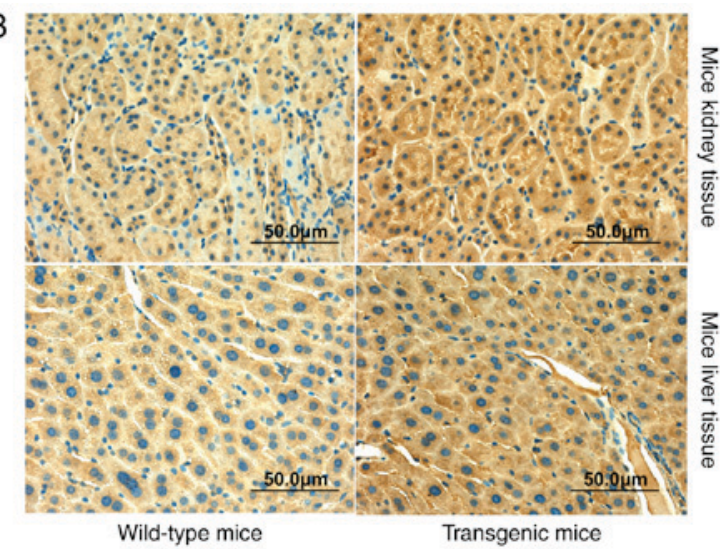

Figure 2. Identification of Nedd4-2 neddylation. (A) Co-immunoprecipitation in kidney and liver of mice demonstrated that interaction was weaker in kidney and stronger in liver. (B) Immunohistochemistry showed that Nedd8 protein expression is higher in both the liver and kidney of transgenic mice compared with wild-type mice; scale bar, $50 \mu \mathrm{m}$. Nedd, neural precursor cell expressed developmentally downregulated; TG, transgenic; WT, wild-type.

wild-type control tissues. These data suggested that 20-HETE may reduce the neddylation of Nedd4-2 in kidney, which may lead to increased expression of Nedd4-2, and that 20-HETE may increase neddylation of Nedd4-2 in liver, resulting in decreased expression of Nedd4-2 in mice.

Expression of Senp8 in CYP4F2 transgenic mice and wild-type mice. To investigate why 20-HETE regulated Nedd4-2 expression contrary to its neddylation status, expression of the deneddylation enzyme Senp8 was examined in
CYP4F2 transgenic and in wild-type mice. Western blotting demonstrated that Senp8 expression was higher in the kidney of transgenic mice compared with wild-type control tissues, and Senp8 expression was lower in transgenic liver tissues compared with wild-type (Fig. 3A). Similar Senp8 protein expression results were obtained from western blotting (Fig. 3B). These data implied that 20-HETE may affect Senp8 expression, which may increase the dissociation of Nedd8 from Nedd4-2 in kidney and decrease the dissociation of Nedd8 from Nedd4-2 in liver, resulting in the downregulation 
A

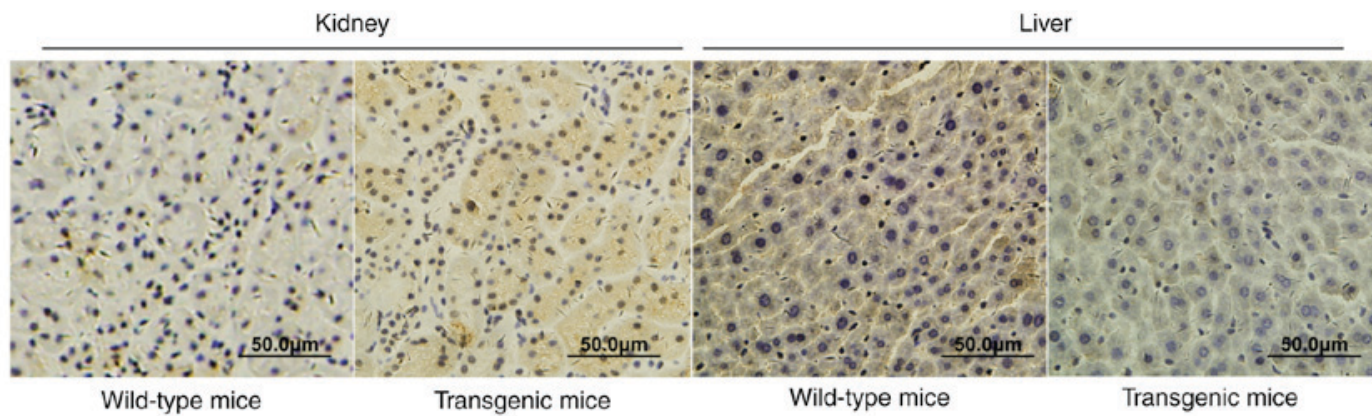

B
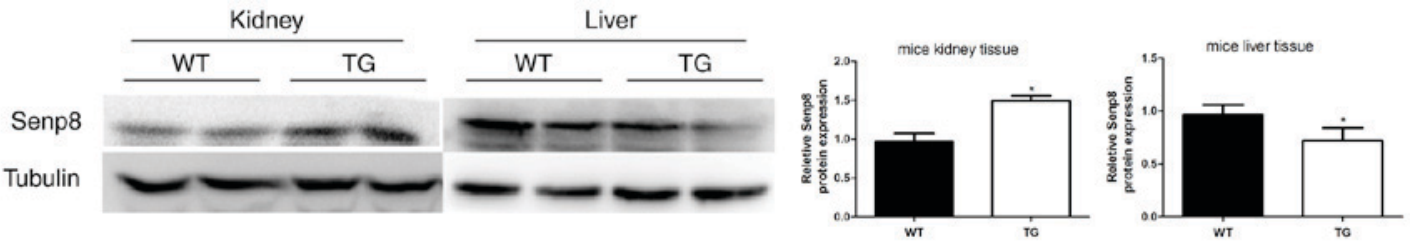

Figure 3. Expression of Senp8 in TG and WT mouse tissues. (A) Immunohistochemical analyses demonstrated that the expression of Senp8 was increased in transgenic mouse kidney, and decreased in transgenic mouse liver compared with their respective wild-type controls; $\mathrm{n}=6$; scale bar, 50 $\mu$ m. (B) Western blotting demonstrated that the expression of Senp8 was higher in kidney of transgenic mice than wild-type control kidney, and Senp8 expression was lower in transgenic mouse liver compare with expression in wild-type controls; all experiments were performed three times independently; $\mathrm{n}=6$ (the tissues in these 3 independent experiments were from 6 mice including 3 TG mice and 3 WT mice); ${ }^{*} \mathrm{P}<0.05$ vs. WT kidney; ${ }^{*} \mathrm{P}<0.05$ vs. WT liver. Senp8, sentrin-specific protease 8 ; TG, transgenic; WT, wild-type.

of Nedd4-2 neddylation in kidney and the upregulation of Nedd4-2 neddylation in liver.

Effects of 20-HETE on neddylation of Nedd4-2. To verify the aforementioned ex vivo results, which indicated that differential Nedd4-2 expression may be linked to neddylation status in kidney and liver of transgenic mice, M1 and NCTC1469 cells were treated with 20-HETE. Co-IP analysis demonstrated that 20-HETE treatment resulted in less binding of Nedd8 to Nedd4-2 in M1 kidney cells and increased binding in NCTC1469 liver cells compared with the respective untreated controls (Fig. 4A). Furthermore, western blotting demonstrated that Nedd4-2 expression was higher in 20-HETE-treated M1 cells and lower in 20-HETE-treated NCTC1469 cells compared with untreated controls (Fig. 4B). To determine the reason for this inverse effect of 20-HETE on neddylation of Nedd4-2, Senp8 protein expression was determined in the same tissues. The results showed that Senp8 expression was in line with Nedd4-2 expression (Fig. 4B). In addition, the effects of 20-HETE on Nedd4-2 were eliminated by co-culturing cell with the neddylation inhibitor MLN4924, which demonstrated that the 20-HETE-induced increases of Nedd4-2 and Senp8 protein expression levels in M1 cells were blocked by MLN4924, and the 20-HETE-induced decreases of Nedd4-2 and Senp8 expression were restored by MLN4924 in NCTC1469 cells (Fig. 4C). Therefore, these results suggested that 20-HETE may positively regulate Nedd4-2 expression through the neddylation pathway in kidney and negatively regulate Nedd4-2 expression in liver. This regulation of 20-HETE performed partly through its effect on the neddylation process.

\section{Disccusion}

A previous study suggested that the most well-known function of neddylation is to enhance E3 ligase activity (23).
The ubiquitination activity of E3 ligases depends in part on neddylation. The CRL family was the first identified and well-studied substrate of neddylation $(14,24)$. Covalent binding of Nedd8 to a conserved lysine in the C-terminal domain stimulates CRL ubiquitination activity and prevents them from binding to the structural-based inhibitor cullin-associated Nedd8-dissociated protein 1 (25). However, the function of Nedd8 covalent conjugation is not only to activate, but also to confer an intrinsic instability (26). Chung et al demonstrated that neddylated cullin proteins are recycled and the conjugated Nedd8 is removed by CSN isopeptidase; otherwise, they are efficiently degraded (27). Thus, the neddylation/deneddylation cycle provides a way to maintain normal cellular levels of activated E3 ubiquitin ligase and to prevent excessive ubiquitin ligase activity (28). Nedd4-2 acts as an E3 ubiquitin ligase of the HECT-type ubiquitin ligase family and has potential Nedd8 covalent conjugation (15).

Neddylation of Nedd4-2 has not been reported to date. In regard to the regulation of Nedd4-2 function, serum/glucocorticoid-regulated kinase 1 has been reported to phosphorylate serine residues 222 and 328 of Nedd4-2, which promoted its binding to 14-3-3 proteins, and consequently inhibited the interaction with its substrates, such as NKCC2 and ENaC (29). It also has been reported that Nedd4-2 is controlled by auto-ubiquitination; however, it may weakly bind to the PY motif in its HECT domain through its WW domain, which suggested that this intramolecular interaction may inhibit its auto-ubiquitination (30). Using co-IP, the present study identified an interaction of Nedd8 with Nedd4-2 in kidney and liver tissues of mice, as well as in M1 and NCTC1469 cell lines. To the best of our knowledge, this is the first preliminary report to demonstrate that Nedd4-2 was modified by Nedd8. Consistent with this, neddylation of Smurf1, such as with HECT-like ligase, has been reported (15). In addition, the data from comparisons between $C Y P 4 F 2$ transgenic mice and wild-type 
A

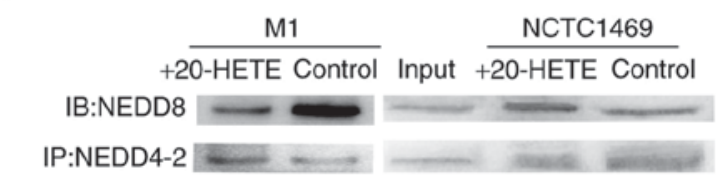

B
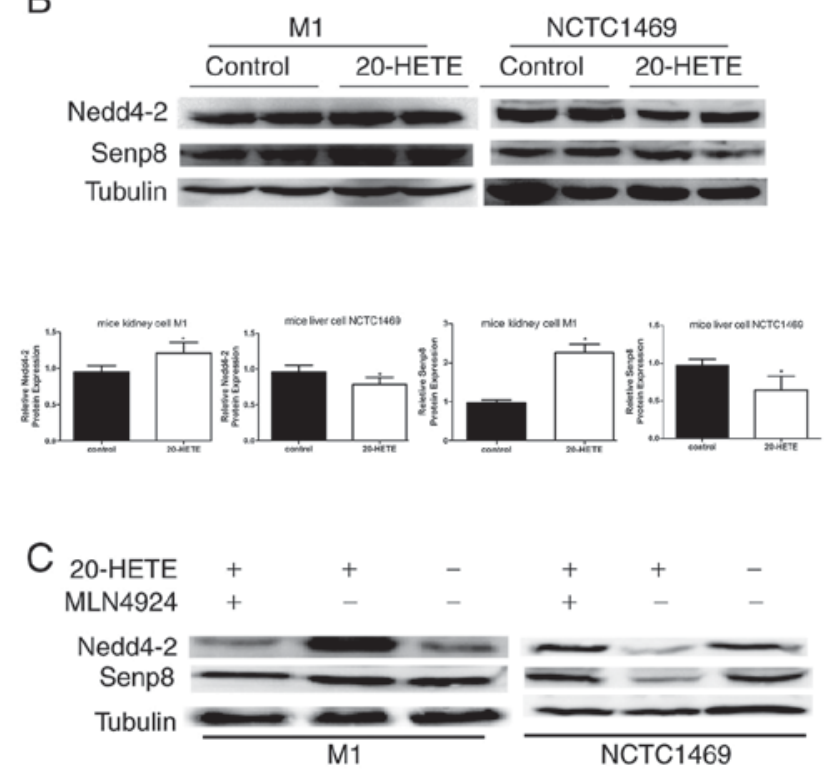

Figure 4. 20-HETE affects the neddylaton of Nedd4-2 in cell culture through Senp8. (A) In M1 mouse kidney cells, Nedd4-2 was less modified by Nedd8 following addition of 20-HETE to the culture medium, whereas the opposite was observed in NCTC1469 mouse liver cells. (B) Western blotting in cell cultures demonstrated that 20-HETE treatment promoted the expression of Senp8 in M1 cells, but inhibited its expression in NCTC1469, which may result in a notable change in Nedd4-2 expression. All experiments were performed three times independently; $\mathrm{n}=6$ (the tissues in these 3 independent experiments were from 6 mice including $3 \mathrm{TG}$ mice and $3 \mathrm{WT}$ mice); ${ }^{\mathrm{P}} \mathrm{P}<0.05$ vs. control; ${ }^{*} \mathrm{P}<0.05$ vs. control. (C) Recovery experiments: In M1 cells treated with 20-HETE alone, the expression level of Nedd4-2 and Senp8 were upregulated, whereas when both MLN4924 and 20-HETE were added, the expression of Nedd4-2 and Senp8 were recovered, compared with untreated control; the opposite results were observed in NCTC1469 cells. 20-HETE, 20-hydroxyeicosatetraenoic acid; Nedd4-2, neural precursor cell expressed developmentally downregulated 4-like, E3 ubiquitin-protein ligase; Senp8, sentrin-specific protease 8 .

mice demonstrated that 20-HETE reduced the neddylation of Nedd4-2 in kidney, leading to probably increased expression of Nedd4-2, and that 20-HETE increased the neddylation of Nedd4-2 in liver, which probably resulted in decreased expression of Nedd4-2 in mice. To verify 20-HETE positively regulated Nedd4-2 in kidney and negatively regulated Nedd4-2 in liver, expression of the deneddylation enzyme Senp8 was examined between transgenic mice and wide-type mice. Senp8, also known as DCN1, was reported more efficient to hyper-neddylated cullins (cullins with more than one combine site of nedd8) and non-cullins compared to mono-neddylated ones (cullins with only one combine site of nedd8) $(17,23)$ The results revealed that Senp8 expression was higher in kidney and lower in liver of transgenic mice compared with wild-type mice, which was similar to the expression of Nedd4-2. Notably, cell culture experiments indicated that the effects of 20-HETE on Nedd4-2 and Senp8 expression were recovered by treatment with the inhibitor MLN4924, which indicated that 20-HETE regulation of Nedd4-2 and Senp8 was at least partly dependent on neddylation modification. Therefore, it was inferred that increased levels of Senp8 dissociated more Nedd8 from Nedd4-2 in kidney to interfere with self-ubiquitination and degradation, and that the opposite occurred in the liver.

Notably, 20-HETE may function in a tissue-specific manner in the kidney and liver: The present study demonstrated that 20-HETE increased the expression of Nedd4-2 and Senp8 in kidney and decreased their expression levels in the liver. In addition, 20-HETE decreased the neddylation of Nedd4-2 in kidney and increased Nedd4-2 neddylation in the liver. Similarly, previous studies have demonstrated tissue-specific expression in the vascular system. On the one hand, 20-HETE was reported to be a vasoconstrictor in renal and cerebral arteries through activation of the protein kinase $\mathrm{C}$ or mitogen-activated protein kinase pathway (31). In addition, 20-HETE may also directly affect the L-type $\mathrm{Ca}^{2+}$ channels on the cell membrane to increase the concentration of intracellular $\mathrm{Ca}^{2+}$, thus triggering vasoconstriction (32). In addition to the vascular system, Nowicki et al have reported that 20-HETE inhibited a cAMP-dependent pathway in the kidney of rats that consumed a potassium-deficient diet (33). By contrast, results from our previous study demonstrated that 20-HETE activated the cAMP pathway in the liver of $C Y P 4 F 2$ transgenic mice (34). In blood pressure regulation, 20-HETE exhibited both prohypertensive and antihypertensive actions through vasoconstriction and natriuresis. This dual role was also evident in or CYP4F2 transgenic mice: 20-HETE had a prohypertensive role in the transgenic mice fed a normal salt diet, whereas 20-HETE exhibited an antihypertensive role by promoting natriuresis in transgenic mice fed a high salt diet (4). This demonstrated that 20-HETE serves different roles in various tissues. The role of 20-HETE is extensive and complicated. These data may provide an insight into the function of 20-HETE on neddylation modification of Nedd4-2. 20-HETE not only participated in blood pressure regulation but was also involved in tumor formation and progression (35), kidney diseases (36) and plasma glucose regulation (34). The present study demonstrated that 20-HETE regulates the expression of Nedd4-2 and Senp8 positively in kidney and negatively in liver. One possible explanation for the differential regulation is that 20-HETE may activate different pathways in different tissues, as 20-HETE has high lipid solubility and is able to freely pass through the cell membrane and exert multiple functions (37). Further examinations of 20-HETE-induced pathways in kidney and liver are required. Another possible explanation is that 20-HETE may have its own receptor that may be distributed differently in various tissues. Recently, several studies have sought to identify a 20-HETE receptor $(38,39)$, which may help to explain the regulation of 20-HETE in kidney and liver.

In summary, the present study demonstrated that 20-HETE upregulated Senp8 expression, which enhanced the deneddylation of Nedd4-2 and interfered with Nedd4-2 degradation, and subsequently resulted in an increased expression level of Nedd4-2 in kidney, and that the opposite effects occured in the liver. The mechanisms by which 20 -HETE positively regulated Nedd4-2 in kidney and negatively regulated Nedd4-2 in the liver remain to be fully understood, but the regulation of 20-HETE between neddylation and deneddylation of Nedd4-2 may be involved. 


\section{Acknowledgements}

This study was supported by The National Natural Science Foundation of China (grant nos. 81270343 and 31571198) and The Outstanding Scientific Fund of Shengjing Hospital (grant no. 201601).

\section{References}

1. Medhora M, Chen Y, Gruenloh S, Harland D, Bodiga S, Zielonka J, Gebremedhin D, Gao Y, Falck JR, Anjaiah S and Jacobs ER: 20-HETE increases superoxide production and activates NAPDH oxidase in pulmonary artery endothelial cells. Am J Physiol Lung Cell Mol Physiol 294: L902-L911, 2008.

2. Pratt PF, Falck JR, Reddy KM, Kurian JB and Campbell WB: 20-HETE relaxes bovine coronary arteries through the release of prostacyclin. Hypertension 31: 237-241, 1998.

3. Liu X, Zhao Y, Wang L, Yang X, Zheng Z, Zhang Y, Chen F and Liu H: Overexpression of cytochrome P450 4F2 in mice increases 20-hydroxyeicosatetraenoic acid production and arterial blood pressure. Kidney Int 75: 1288-1296, 2009.

4. Wu J, Liu X, Lai G, Yang X, Wang L and Zhao Y: Synergistical effect of 20-HETE and high salt on NKCC2 protein and blood pressure via ubiquitin-proteasome pathway. Hum Genet 132: 179-187, 2013.

5. Harvey KF, Dinudom A, Cook DI and Kumar S: The Nedd4-like protein KIAA0439 is a potential regulator of the epithelial sodium channel. J Biol Chem 276: 8597-8601, 2001.

6. Rougier JS, van Bemmelen MX, Bruce MC, Jespersen T, Gavillet B, Apothéloz F, Cordonier S, Staub O, Rotin D and Abriel H: Molecular determinants of voltage-gated sodium channel regulation by the Nedd4/Nedd4-like proteins. Am J Physiol Cell Physiol 288: C692-C701, 2005.

7. Kamynina E, Debonneville C, Bens M, Vandewalle A and Staub O: A novel mouse Nedd4 protein suppresses the activity of the epithelial Na+ channel. FASEB J 15: 204-214, 2001.

8. Laedermann CJ, Cachemaille M, Kirschmann G, Pertin M, Gosselin RD, Chang I, Albesa M, Towne C, Schneider BL, Kellenberger S, et al: Dysregulation of voltage-gated sodium channels by ubiquitin ligase NEDD4-2 in neuropathic pain. J Clin Invest 123: 3002-3013, 2013.

9. Hellwinkel OJ, Asong LE, Rogmann JP, Sültmann H, Wagner C, Schlomm $\mathrm{T}$ and Eichelberg C: Transcription alterations of members of the ubiquitin-proteasome network in prostate carcinoma. Prostate Cancer Prostatic Dis 14: 38-45, 2011.

10. Kito Y, Bai J, Goto N, Okubo H, Adachi Y, Nagayama T and Takeuchi T: Pathobiological properties of the ubiquitin ligase Nedd4L in melanoma. Int J Exp Pathol 95: 24-28, 2014.

11. Wen H, Lin R, Jiao Y, Wang F, Wang S, Lu D, Qian J, Jin L and Wang X: Two polymorphisms in NEDD4L gene and essential hypertension in Chinese Hans-a population-based case-control study. Clin Exp Hypertens 30: 87-94, 2008.

12. Whitby FG, Xia G, Pickart CM and Hill CP: Crystal structure of the human ubiquitin-like protein NEDD8 and interactions with ubiquitin pathway enzymes. J Biol Chem 273: 34983-34991, 1998.

13. Huang DT, Hunt HW, Zhuang M, Ohi MD, Holton JM and Schulman BA: Basis for a ubiquitin-like protein thioester switch toggling E1-E2 affinity. Nature 445: 394-398, 2007.

14. Pan ZQ, Kentsis A, Dias DC, Yamoah K and Wu K: Nedd8 on cullin: Building an expressway to protein destruction. Oncogene 23: 1985-1997, 2004.

15. Xie P, Zhang M, He S, Lu K, Chen Y, Xing G, Lu Y, Liu P, Li Y, Wang S, et al: The covalent modifier Nedd8 is critical for the activation of Smurf1 ubiquitin ligase in tumorigenesis. Nat Commun 5: 3733, 2014.

16. Boh BK, Smith PG and Hagen T: Neddylation-induced conformational control regulates cullin RING ligase activity in vivo. J Mol Biol 409: 136-145, 2011.

17. Goldenberg SJ, Cascio TC, Shumway SD, Garbutt KC, Liu J, Xiong Y and Zheng N: Structure of the Cand1-Cul1-Roc1 complex reveals regulatory mechanisms for the assembly of the multisubunit cullin-dependent ubiquitin ligases. Cell 119: 517-528, 2004.

18. Kapelari B, Bech-Otschir D, Hegerl R, Schade R, Dumdey R and Dubiel W: Electron microscopy and subunit-subunit interaction studies reveal a first architecture of COP9 signalosome. J Mol Biol 300: 1169-1178, 2000
19. Wei W, Guo H, Liu X, Zhang H, Qian L, Luo K, Markham RB and Yu XF: A first-in-class NAE inhibitor, MLN4924, blocks lentiviral infection in myeloid cells by disrupting neddylation-dependent Vpx-mediated SAMHD1 degradation. J Virol 88: 745-751, 2014

20. Sarantopoulos J, Shapiro GI, Cohen RB, Clark JW, Kauh JS, Weiss GJ, Cleary JM, Mahalingam D, Pickard MD, Faessel HM, et al: Phase I study of the investigational NEDD8-activating enzyme inhibitor pevonedistat (TAK-924/MLN4924) in patients with advanced solid tumors. Clin Cancer Res 22: 847-857, 2016.

21. Shah JJ, Jakubowiak AJ, O'Connor OA, Orlowski RZ, Harvey RD, Smith MR, Lebovic D, Diefenbach C, Kelly K, Hua Z, et al: Phase I study of the novel investigational NEDD8-activating enzyme inhibitor Pevonedistat (MLN4924) in patients with relapsed/refractory multiple myeloma or lymphoma. Clin Cancer Res 22: 34-43, 2016.

22. Livak KJ and Schmittgen TD: Analysis of relative gene expression data using real-time quantitative PCR and the 2(-Delta Delta C(T)) method. Methods 25: 402-408, 2001.

23. Bornstein G and Grossman C: COP9-Signalosome deneddylase activity is enhanced by simultaneous neddylation: Insights into the regulation of an enzymatic protein complex. Cell Div 10: 5, 2015.

24. Wu K, Chen A and Pan ZQ: Conjugation of Nedd8 to CUL1 enhances the ability of the ROC1-CUL1 complex to promote ubiquitin polymerization. J Biol Chem 275: 32317-32324, 2000.

25. Kurz T, Ozlü N, Rudolf F, Luke B, Hofmann K, Hyman AA, Bowerman B and Peter M: The conserved protein DCN-1/Dcn1p is required for cullin neddylation in C. Elegans and S. Cerevisiae. Nature 435: 1257-1261,2005.

26. Kurz T, Chou YC, Willems AR, Meyer-Schaller N, Hecht ML, Tyers M, Peter M and Sicheri F: Dcn1 functions as a scaffold-type E3 ligase for cullin neddylation. Mol Cell 29: 23-35, 2008.

27. Chung D and Dellaire G: The Role of the COP9 signalosome and neddylation in DNA damage signaling and repair. Biomolecules 5: 2388-2416, 2015.

28. Wu JT, Lin HC, Hu YC and Chien CT: Neddylation and deneddylation regulate $\mathrm{Cul1}$ and $\mathrm{Cul} 3$ protein accumulation. Nat Cell Biol 7: 1014-1020, 2005.

29. Chandran S, Li H, Dong W, Adams C, Alexandrova L, Chien A Hallows KR and Bhalla V: Neural precursor cell-expressed developmentally down-regulated protein 4-2 (Nedd4-2) regulation by 14-3-3 protein binding at canonical serum and glucocorticoid kinase 1 (SGK1) phosphorylation sites. J Biol Chem 286: 37830-37840, 2011.

30. Bruce MC, Kanelis V, Fouladkou F, Debonneville A, Staub O and Rotin D: Regulation of Nedd4-2 self-ubiquitination and stability by a PY motif located within its HECT-domain. Biochem J 415: 155-163, 2008.

31. Singh H and Schwartzman ML: Renal vascular cytochrome P450-derived eicosanoids in androgen-induced hypertension. Pharmacol Rep 60: 29-37, 2008.

32. Mulligan SJ and MacVicar BA: Calcium transients in astrocyte endfeet cause cerebrovascular constrictions. Nature 431: 195-199, 2004.

33. Nowicki S, Chen SL, Aizman O, Cheng XJ, Li D, Nowicki C, Nairn A, Greengard P and Aperia A: 20-Hydroxyeicosa-tetraenoic acid (20 HETE) activates protein kinase C. Role in regulation of rat renal Na+,K+-ATPase. J Clin Invest 99: 1224-1230, 1997.

34. Lai G, Wu J, Liu X and Zhao Y: 20-HETE induces hyperglycemia through the cAMP/PKA-PhK-GP pathway. Mol Endocrinol 26: 1907-1916, 2012

35. Yu W, Chen L, Yang YQ, Falck JR, Guo AM, Li Y and Yang J: Cytochrome P450 $\omega$-hydroxylase promotes angiogenesis and metastasis by upregulation of VEGF and MMP-9 in non-small cell lung cancer. Cancer Chemother Pharmacol 68: 619-629, 2011.

36. Park F, Sweeney WE, Jia G, Roman RJ and Avner ED: 20-HETE mediates proliferation of renal epithelial cells in polycystic kidney disease. J Am Soc Nephrol 19: 1929-1939, 2008.

37. Escalante B, Erlij D, Falck JR and McGiff JC: Cytochrome P450-dependent arachidonate metabolites affect renal transport in the rabbit. J Cardiovasc Pharmacol 22 (Suppl 2): S106-S108, 1993.

38. Ward NC, Chen K, Li C, Croft KD and Keaney JF Jr: Chronic activation of AMP-activated protein kinase prevents 20-hydroxyeicosatetraenoic acid-induced endothelial dysfunction. Clin Exp Pharmacol Physiol 38: 328-333, 2011.

39. Sodhi K, Wu CC, Cheng J, Gotlinger K, Inoue K, Goli M, Falck JR, Abraham NG and Schwartzman ML: CYP4A2-induced hypertension is 20-hydroxyeicosatetraenoic acid- and angiotensin II-dependent. Hypertension 56: 871-878, 2010. 